\title{
DISTORTIONS IN COMPACT STEEP-SPECTRUM RADIO SOURCES ${ }^{+}$
}

P.N. Wilkinson, R.E. Spencer.

Nuffield Radio Astronomy Laboratories, Jodrell Bank

A.C.S. Readhead and T.J. Pearson

Owens Valley Observatory, California Institute of Technology

R.S. Simon

Naval Research Laboratory, Washington, D.C.

The size, and therefore the importance, of the population of compact, steep-spectrum, radio sources has only recently been recognised ${ }^{1,2}$. While it is now clear that the extended steep-spectrum sources are powered by a pair of, originally anti-parallel, beams which transport energy to the outer lobes some $10^{5}-10^{6}$ parcsec away, our understanding of the compact steep-spectrum sources is almost nil. This is largely because our radio maps have not had high enough resolution to show their structures in any detail. However 1.67 and $5 \mathrm{GHz}$ MERLIN observations (resolutions 0!'25 and 0!'1) of the 220 steep-spectrum 3CR sources whose LAS is $\leqslant 2$ " have now allowed us to classify their structures, at least in broad terms. These MERLIN maps, and recent VLBI maps, show that while there is a wide range of structures - from col inear doubles to amorphous "blobs" - the "peculiar" structures are strongly concentrated in the objects whose optical counterparts are called QSO's.

The 3CR QSO's which we include in this, as yet subjectively defined "peculiar" class, are 3C48 (see Fig.1) 3C147 (see Fig.2 and Preuss et al. and Simon et al.); 3C216 (no map available) 3C309.1 (see Fig.3); 3C343 (see Fanti et al.), 3C380 (see Fig.4); 3C454 (see Cawthorne) $3 \mathrm{C} 380$ is included although its LAS is $>2$ " because the brightest components at $5 \mathrm{GHz}$ do fall within 2" of each other. About half these $3 \mathrm{CR}$ sources are QSOs and about half are galaxies but among the galaxies only 3C299 clearly has a "peculiar"structure - most of the rest of them are rather colinear and undistorted (see Fanti et al.). While a few of these sources have not yet been mapped in detail the separation between QSOs and galaxies, in this specific morphological respect, is clear-cut.

What does this mean? First of all it is important to realise that the projected sizes of all these radio sources are of sub-galactic (i.e. a few tens of $\mathrm{kpc}$ ) dimensions and thus the beams could well be propagating through interstellar gas throughout most of their paths. There is strong evidence, from observations of a few steep-spectrum radio cores in nearby galaxies (e.g. ref 3 and van Breugel et al.) that interstellar gas can interact strongly with beams. The pressures in the radio plasma and the emission line clouds in these galaxies are rather similar

+ Discussion on page 418.

25

R. Fanti et al. (eds.), VLBI and Compact Radio Sources, 25-28.

(C) 1984 by the IAU. 


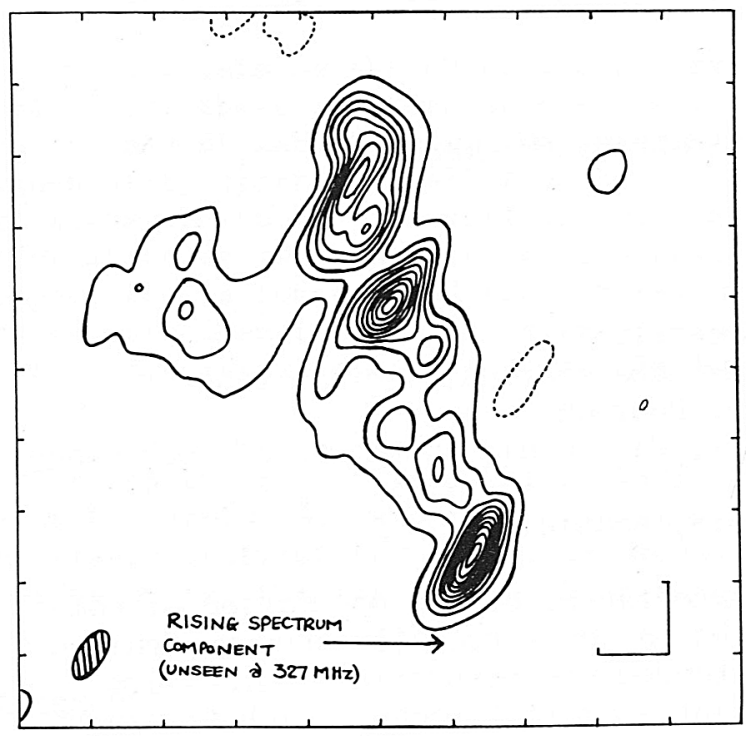

Fig. $1 \quad 3 \mathrm{C} 48: 327 \mathrm{MHz}$ VLBI (Simon et al., in preparation). Scale indicates $0.05 "=150 \mathrm{pc}$ (for $\mathrm{H}_{\mathrm{O}}=100 \mathrm{~km} \mathrm{~s}^{-1} \mathrm{Mpc}^{-1}$ $\mathrm{q}_{\mathrm{O}}=0.5$ )

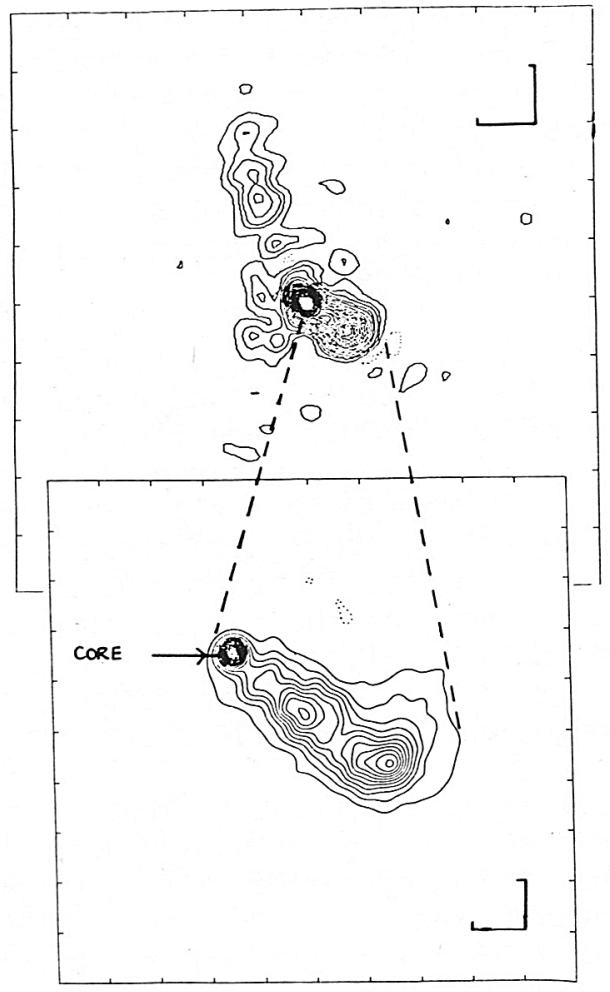

Fig.2 (top) 3C 147

$5 \mathrm{GHz}$ MERLIN (Wilkinson et al., in preparation) Scale indicates $0.2^{\prime \prime}=$ $=740 \mathrm{pc}$.

(bottom) 3C 147

$327 \mathrm{MHz}$ VLBI (Simon et al., in preparation) Scale indicates $0.05^{\prime \prime}=$ $=185 \mathrm{pc}$ 

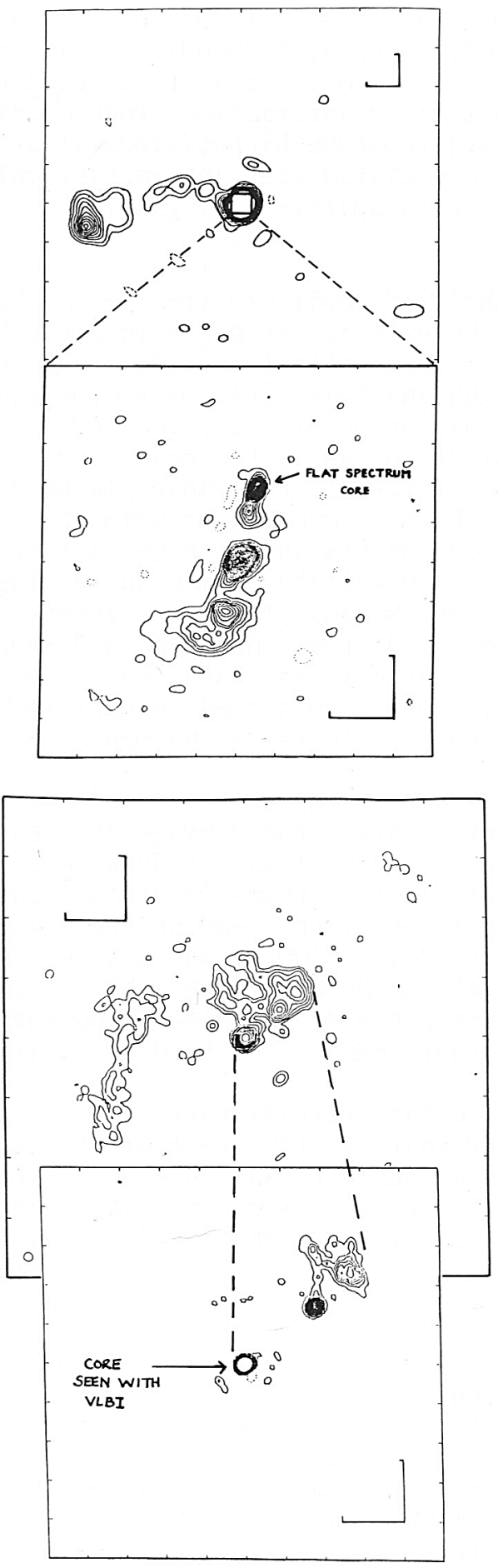

Fig. 3 (top) 3C $309.1: 5 \mathrm{GHz}$ MERLIN (Wilkinson et al., in preparation. Scale indicates $0.2^{\prime \prime}=840 \mathrm{pc}$.

N.B.: there is also low brightness emission to the west of the nucleus (see Proc. IAU Symposium $\mathrm{N}_{\mathrm{O}} \cdot 97$, p152,Fig.6)

(bottom) 3C $309.1: 1.67 \mathrm{GHz}$ VLBI (Wilkinson et al., in preparation). Scale indicates $0.02=84 \mathrm{pc}$.
Fig. 4 (top) 3C $380: 1.67 \mathrm{GHz}$ MERLIN+VLBI (Wilkinson et a1., in preparation). Scale indicates $1^{\prime \prime}=4 \mathrm{kpc}$.

(bottom) 3C $380: 5 \mathrm{GHz}$ MERLIN (Wilkinson et al., in preparation). Scale indicates $0.5 "=" \mathrm{kpc}$.

N.B.: there are other low brightness features (see Proc. IAU Symposium $\mathrm{N}_{\mathrm{O}} \cdot 97$, p157 Fig.10) 
$\left(10^{-8} \cdot 5\right.$ dyne $\left.\mathrm{cm}^{-2}\right)$, the optical 1 ine emission is usually brightest near radio knots or hotspots and the beams often appear to bend near bright emission-1 ine clouds. If we are to ascribe the much greater distortions in these highly radio-luminous QSOs to similar interactions then clearly the energy density of the interstellar gas must be higher. Thus it is interesting that many QSO's seem to be associated with interacting galaxy systems and some of them are associated with spirals i.e. gas-rich objects ${ }^{4}$.

The specific case of the low redshift QSO 0351+026 (ref 5) may be particularly important in this respect because it "strongly resembles" (see ref 6) one of our QSOs, 3C48. 0351+026 is clearly a pair of gas-rich galaxies undergoing a violent interaction and this gives us a clue that perhaps the other QSO's with peculiar radio structures are located in similar interacting/merging systems. Extended gas with a mean density of $>10^{-23.5} \mathrm{gm} \mathrm{cm}^{-3}$ and a bulk velocity relative to the radio plasma of $2500 \mathrm{~km} \mathrm{~s}^{-1}$ would exert a ram pressure which is comparable with the radio plasma pressure of the $\mathrm{kpc}$-scale components in these QSO's i.e. $\left(10^{-}(8 \pm 1)\right.$ dyne $\mathrm{cm}^{-2}$. Such energetic gas may well be common in merging systems. Thus, if the beams are very light, strong plasma/gas interactions may well be common in QSO's. Severe distortions or even disruption of the beams could occur in highly energetic gas and clearly computer simulations using 3-D hydrodynamic codes are needed to explore the range of circumstances under which these effects can happen.

The properties of the gas within $\sim 1 \mathrm{kpc}$ of the nucleus in QSO's are very uncertain. However in nearby spiral galaxies the average gas density in this region is usually at least an order of magnitude higher than it is further out. Thus the morphological features in some of our VLBI maps e.g. the non-colinear feature in 3C48, the bend at the end of the 'jet' in 3C147, the sharp apparent bend in 3 C 309.1, may also be due to interactions with surrounding material on the $0.1-1 \mathrm{kpc}$ scale. This is despite the fact that the radio plasma pressure in these components is typically two orders of magnitude higher than on the $1-10 \mathrm{kpc}$ scale.

To summarise: QSOs preferentially contain very distorted radio sources compared with radio galaxies. By analogy with the compact steepspectrum cores in a few nearby galaxies we suspect that this is due to an interaction with energetic interstellar gas. A circumstantial case can be made that this energetic gas is associated with mergers of gasrich galaxies.

\section{REFERENCES}

N.B. Named references are to papers in this volume

1) Kapahi, V.K. Astron.Astrophys.Supp1., 43, 381 (1981)

2) Peacock, J. and Wall, J., M.N.R.A.S. 198,843 (1982)

3) van Breuge1, W. and Heckman, T., Proc.IAU Symposium No.97, p.61

4) Hutchings, J.B. and Campbe11, B., Nature 303, 584 (1983)

5) Bothun, G.D. et al. Astron.J. 87, 1621 (1982)

6) Balick, B. and Heckman, T. Ast rophys.J., 265, L1 (1983) 